\title{
ENGAGEMENT DE VOLUNTARIOS. UN ESTUDIO COMPARATIVO
}

\section{VOLUNTEERS' ENGAGEMENT. A COMPARATIVE STUDY}

\author{
Yamila Fernanda Silva Peralta \\ Facultad de Psicología \\ Universidad Nacional de Mar del Plata \\ Consejo Nacional de Investigaciones Científicas y \\ Técnicas \\ Mar del Plata, Buenos Aires, Argentina \\ yamilasilvaperalta@gmail.com \\ Lorena Verónica Caracciolo \\ Facultad de Psicología \\ Universidad Nacional de Mar del Plata \\ Mar del Plata, Buenos Aires, Argentina \\ lolica81@gmail.com
}

\author{
Sergio Gastón Arias \\ Facultad de Psicología \\ Universidad Nacional de Mar del Plata \\ Mar del Plata, Buenos Aires, Argentina \\ sigma87@hotmail.com
}

\author{
Juan Pablo Vega \\ Facultad de Psicología \\ Universidad Nacional de Mar del Plata \\ Mar del Plata, Buenos Aires, Argentina \\ pablovega03@hotmail.com
}

\author{
María Emilia Rompato \\ Facultad de Psicología \\ Universidad Nacional de Mar del Plata \\ Mar del Plata, Buenos Aires, Argentina \\ merompato@gmail.com
}

Fecha de recepción: 22/03/2019 - Fecha de aprobación: 26/06/2019

\section{RESUMEN}

Se presenta una investigación descriptiva/comparativa que tiene como finalidad conocer niveles de engagement en voluntarios universitarios y voluntarios de organizaciones asociativas. Se administró el Utrecht Work Engagement Scale que mide las escalas vigor, absorción, dedicación y el nivel de engagement. La muestra de esta investigación fue intencional, no probabilística, compuesta por: (a) voluntarios de la Universidad Nacional de Mar del Plata (UNMDP) que han participado en los últimos 4 años en proyectos de extensión y voluntariado universitario y (b) voluntarios de organizaciones asociativas de Mar del Plata. Fueron calculados descriptivos básicos y aplicados estadísticos no paramétricos con la finalidad de comparar grupos de voluntarios. Los resultados muestran diferencias críticas cuando se agrupa por sexo en dedicación y vigor, por nivel de instrucción/formación en vigor y engagement (puntaje total). No se encontraron niveles críticos agrupando por tipo de voluntariado, por edad ni por antigüedad como voluntario.

PALABRAS CLAVE: Engagement; Voluntariado; Voluntariado universitario; Tercer Sector.

\footnotetext{
“Visión de Futuro" Año 17, Volumen N²4 N¹, Enero - Junio 2020 - Pág. 131 - 148

URL de la Revista: http://visiondefuturo.fce.unam.edu.ar/index.php/visiondefuturo/index

URL del Documento: https://visiondefuturo.fce.unam.edu.ar/index.php/visiondefuturo/issue/view/17

ISSN 1668 - 8708 - Versión en Línea

E-mail: revistacientifica@fce.unam.edu.ar
}

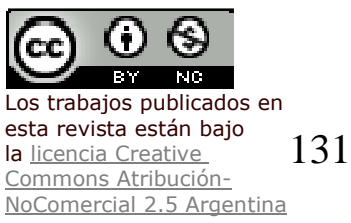




\section{ABSTRACT}

A descriptive/comparative research is presented with the aim of knowing engagement levels in university volunteers and associative organizations volunteers' groups. The Utrecht Work Engagement Scale was administered; it measures vigor, absorption and dedication scales and engagement levels. The sample of this research was intentional non-probabilistic, composed of (a) volunteers from the National University of Mar del Plata (UNMDP) participating during the last 4 years in extension and volunteering projects and (b) associative organizations volunteers from Mar del Plata. Basic descriptive were calculated and non-probabilistic statistics were applied to compare volunteering groups. Results show critical differences between sex in dedication and vigor, between training levels in vigor and engagement (total score). No critical levels were found between volunteering type, age and seniority as a volunteer.

KEY WORDS: Engagement; Volunteering; University volunteering; Third Sector.

\section{INTRODUCCIÓN}

La investigación, de carácter descriptiva, tiene como finalidad conocer los niveles de engagement en grupos de voluntarios de la Universidad Nacional de Mar del Plata (en adelante UNMDP) y voluntarios de organizaciones asociativas de la misma ciudad. Específicamente se busca: (a) describir los niveles de engagement en ambos grupos de voluntarios, (b) comparar los niveles de vigor, dedicación y absorción (escalas del engagement) en ambos grupos de voluntarios y (c) evaluar niveles críticos de asociación entre engagement y variables sociodemográficas (sexo, edad, nivel de instrucción/formación y antigüedad como voluntario).

Esta investigación supone la incursión en el estudio de competencias sociales que son decisivas para un mundo que requiere de transformaciones sociales, de valores, de compromiso, tolerancia, respeto por el otro y de capacidad de entrega altruista y solidaria.

Se enmarca desde la Psicología Positiva que coloca el énfasis en factores positivos entre los cuales se halla el engagement. Este factor, por sus características, resulta decisivo en el marco de actividades de voluntariado, segundo gran eje de esta investigación, las que se definen por no ser lucrativas y demandan un alto nivel de involucramiento y compromiso, resultando cada vez más necesarias en el marco de una sociedad que presenta un alto riesgo psicosocial. Se observa escasez de investigaciones que relacionen el engagement con la actividad del voluntariado, siendo este tipo de actividad fundamental en la promoción 
de una sociedad solidaria y participativa.

\section{DESARROLLO}

\section{Revisión teórica}

Tradicionalmente, las investigaciones que se han centrado en estudiar los factores que afectan a la salud y al bienestar laboral, lo han hecho desde una perspectiva negativa intentando identificar enfermedades, factores de riesgo, datos epidemiológicos, niveles de malestar tales como ansiedad, estrés laboral, etc. Sin embargo, en los últimos años han aumentado los estudios que emplean un enfoque positivo, intentando identificar factores protectores del bienestar profesional y personal del trabajador y explicar por qué hay personas y organizaciones que gozan de mayor energía positiva en relación con el trabajo, así como en la manera de conseguirlo (Díez y Dolan, 2008; Blanch, Sahagún y Cervantes, 2010). Concretamente, las últimas tendencias en el estudio del burnout o estrés laboral crónico han dado un giro hacia el estudio de un factor opuesto: el engagement. Este cambio es debido a un cierto auge de la Psicología Positiva, sobre todo en la última década, concepción que se centra no tanto en las debilidades y las disfunciones del ser humano sino en sus fortalezas y su funcionamiento óptimo (Seligman y Csikszentmihalyi, 2000; GarcíaRenedo, Llorens, Cifre y Salanova, 2006).

El engagement ha sido definido como un estado afectivo positivo, relativamente persistente, de plenitud, que es caracterizado por el vigor, la dedicación y la absorción o concentración en el trabajo (Salanova, Schaufeli, Llorens, Peiró y Grau, 2000; Manzano, 2002; Schaufeli, Martínez, Marques Pinto, Salanova y Bakker, 2002). El vigor se refiere a altos niveles de energía y activación mental en el trabajo, la voluntad y predisposición de invertir esfuerzos y la persistencia, incluso ante las dificultades. La dedicación denota una alta implicación laboral, junto con la manifestación de un alto nivel de significado atribuido al trabajo. Se asocia al entusiasmo, estar orgulloso e inspirado en el trabajo, se caracteriza por un sentimiento de importancia y desafío. La absorción es caracterizada por un estado de concentración, de sentimiento de que el tiempo pasa rápidamente y uno tiene dificultades para desligarse del trabajo, debido a la fuerte sensación de disfrute, realización y concentración experimentados.

Salanova, Schaufeli, Llorens, Peiró y Grau (2000) han encontrado que existiría una tendencia a que la absorción funcione de forma independiente en relación con las dimensiones de vigor y dedicación, lo cual se debería a que la absorción sería más una consecuencia del engagement y no tanto una dimensión del mismo. En consonancia, otros

\footnotetext{
“Visión de Futuro" Año 17, Volumen No 24 N¹, Enero - Junio 2020 - Pág. 131 - 148

URL de la Revista: http://visiondefuturo.fce.unam.edu.ar/index.php/visiondefuturo/index

URL del Documento: https://visiondefuturo.fce.unam.edu.ar/index.php/visiondefuturo/issue/view/17

ISSN 1668 - 8708 - Versión en Línea

E-mail: revistacientifica@fce.unam.edu.ar
} 
autores se refieren a la dedicación y al vigor como el "corazón del engagement" (Lisbona, Morales y Palací, 2009) de la misma manera que el agotamiento y la despersonalización son considerados el núcleo central del burnout. Las investigaciones muestran que se puede establecer una correlación negativa entre las escalas de burnout y engagement: entre agotamiento y vigor y entre cinismo (despersonalización) y dedicación debido a que son escalas que puntúan inversamente (Schaufeli, Martínez, Marques Pinto, Salanova y Bakker, 2002; González-Roma, Schaufeli, Bakker y Lloret, 2006).

Salanova, Schaufeli, Llorens, Peiró y Grau (2000) sostienen que el engagement es un constructo motivacional, ya que posee componentes de activación, energía, esfuerzo y persistencia; por lo demás, está dirigido a la consecución de objetivos. Las personas que experimentan engagement, se muestran enérgicas y eficazmente unidas a sus actividades laborales y se sienten totalmente capaces de responder a las demandas de su puesto de trabajo con absoluta eficacia (Maslach y Leiter, 1997; Salanova, Agut y Peiró, 2005). Las consecuencias del engagement son la actitud positiva hacia el trabajo y hacia la organización (reflejada en la satisfacción laboral, compromiso organizacional y baja intención de abandonar el trabajo), alta motivación para aprender cosas nuevas y asumir nuevos retos en el trabajo, conductas de iniciativa personal, desempeño exitoso en las tareas, una mayor calidad del servicio a los clientes y un aumento de la salud de los empleados, siendo uno de los indicadores, la disminución de quejas psicosomáticas. Otra importante consecuencia del engagement hace referencia a la extrapolación de emociones positivas desde el trabajo a la casa y viceversa, que se plasma en una mejor conciliación de la vida familiar y laboral (Salanova, Schaufeli, Llorens, Peiró y Grau, 2000).

El engagement ha sido estudiado en relación a variables sociodemográficas como edad y sexo (Chiang, Núñez, Martín y Salazar, 2010; Benevides-Pereira, Fraiz de Camargo y Porto-Martins, 2011) no encontrándose asociaciones relevantes, aunque existiría una tendencia a considerar que los niveles altos de engagement son más frecuentes en el sexo masculino y los niveles bajos, en el sexo femenino.

En el encuadre de la investigación, el engagement, entendido como un concepto positivo, es fundamental para entender el sostenimiento de actividades no remunerativas y no obligatorias como el voluntariado (Clary y Snyder, 1999), siendo uno de los factores más importantes a tener en cuenta en las organizaciones no lucrativas (Vecina, Chacón, Sueiro y Barrón, 2012).

En relación con término voluntariado, puede advertirse que ha adquirido diferentes sentidos a lo largo de la historia. El concepto de voluntariado adquiere diferentes formas y significados según el entorno, está muy influenciado por la historia, la política, la religión y la cultura de una región. El voluntariado remite principalmente al carácter de gratuidad y 
libertad con que se ofrece un tiempo personal (Cabezas Gonzales, 2001; Chacón y Vecina, 2002; Cruz Roja Española, 2002; Tapia, 2007; Tapia, 2010), volviéndose inespecífico en cuanto a las características, los objetivos y las modalidades de la actividad realizada en ese tiempo donado gratuitamente. Ello determina la diversidad de definiciones halladas en la literatura. Frecuentemente, se menciona el carácter de solidaridad y compromiso social del voluntariado, así como la contextualización del mismo en organizaciones sin fines de lucro y/o programas de acción social, sosteniéndose que el trabajo del voluntario proviene de un proceso de sensibilización y concienciación, respetando plenamente al individuo o individuos a quienes dirige su actividad y puede trabajar de forma aislada, aunque por lo general actúa en grupo (Vecina, 2001; Chacón y Vecina, 2002). Gutiérrez Resa (1997) define al voluntariado como la prestación voluntaria y solidaria de una parte del propio tiempo y facultades en beneficio de otros ciudadanos que lo necesitan, contextualizada en organizaciones y programas de acción social y sin recibir contraprestaciones económicas.

Siguiendo una definición en sentido amplio, puede pensarse al voluntariado como un continuum en el que se presenta cierta graduación y diferencias existentes entre sus formas más informales y esporádicas hasta las más formales e intensivas (Tang, McBride y Sherraden, 2003) siguiendo una serie de criterios tales como estructura, formalidad, duración, intensidad y remuneración. La idea de continuum enfatiza la unidad del concepto de voluntariado, estableciendo simultáneamente la graduación y diferencias existentes entre sus diferentes modalidades (Davis Smith, 2002).

Las organizaciones asociativas son un ámbito fértil para la actividad de voluntariado. Los últimos años han sido testigos del interés suscitado en torno al amplio abanico de instituciones sociales que actúan fuera de los límites del mercado y del Estado. A pesar de su diversidad, estas organizaciones comparten algunos rasgos comunes como: estar organizadas, ser autónomas, no repartir beneficios, contar con voluntariado y tener carácter privado (Roitter, 2001). Puede advertirse que el voluntariado es uno de los requisitos para la conformación de este tipo de organizaciones (Estébanez, 2005).

Otro ámbito que ofrece la posibilidad de que personas se acerquen a desempeñar de forma voluntaria una serie de actividades, es la universidad. Según postulan Hustinx, Vanhove, Declercq, Hermans y Lammertyn (2005), el voluntariado en la universidad o el voluntariado universitario incluye actividades extracurriculares-espontáneas, no pagadas, orientadas externamente, no limitadas en el tiempo ni en tipo de actividades, teniendo lugar dentro como fuera de la universidad. Arias Careaga (2008) sostiene que la integración de actividades de voluntariado en la universidad puede favorecer la promoción de valores como la solidaridad, el altruismo o la tolerancia, y constituir una ventaja para el futuro profesional

\footnotetext{
“Visión de Futuro" Año 17, Volumen No 24 N¹, Enero - Junio 2020 - Pág. 131 - 148

URL de la Revista: http://visiondefuturo.fce.unam.edu.ar/index.php/visiondefuturo/index

URL del Documento: https://visiondefuturo.fce.unam.edu.ar/index.php/visiondefuturo/issue/view/17

ISSN 1668 - 8708 - Versión en Línea 
del alumno. La misma autora señala que lo que distingue al voluntariado universitario de otros tipos de voluntariado es que se enfatiza el sujeto que realiza la acción frente a qué o sobre quién la realiza, como suele ser habitual en el resto de voluntariados. Según la autora, hay características que diferencian al voluntariado universitario del voluntariado en organizaciones asociativas, entre ellas: la formación de profesionales para la inserción en el mundo laboral, la acción voluntaria como complemento a la formación profesional, el voluntariado entendido como una vía de sensibilización y formación de valores, que no nace directamente del entorno del mundo asociativo y, por último, la dimensión, orientación y tipo de acciones puestos en marcha.

Con respecto a la permanencia en la actividad de voluntariado, Dávila de León y Chacón Fuertes (2004) encontraron que existe una relación directa y negativa significativa entre la motivación de mejora del currículum y el tiempo real de permanencia. Los autores hipotetizan que las personas que han satisfecho su necesidad de adquirir experiencia útil profesionalmente o han conseguido un trabajo remunerado, abandonan la organización. Por otra parte, Vecina, Chacon y Sueiro (2010) sostienen que los voluntarios permanentes presentan niveles mayores de cansancio emocional, de compromiso organizacional y de identidad de rol como voluntarios. También parecen estar más satisfechos con las relaciones de amistad en la organización y tienen mayor intención de permanecer a largo plazo (dos años). Vecina, Chacón, Sueiro y Barrón (2012) afirman que el engagement es una variable crucial para explicar la satisfacción del voluntario, mientras que esta última es la variable que permite explicar la intención de permanecer en la actividad.

\section{Metodología}

Se trata de una investigación descriptiva/comparativa. Se analizaron los niveles de engagement de dos grupos de voluntarios: voluntarios universitarios y de organizaciones asociativas de la ciudad de Mar del Plata.

La muestra de esta investigación es intencional, no probabilística, compuesta por voluntarios de la Universidad Nacional de Mar del Plata (en adelante UNMDP) que han participado en los últimos 4 años en proyectos de voluntariado universitario o proyecto de extensión ( $\mathrm{N}=31$ ) y voluntarios de organizaciones asociativas de la ciudad de Mar del Plata $(\mathrm{N}=56)$. Se trabajó con una muestra de voluntarios que pertenecen a 15 organizaciones diferentes, las cuales se clasificaron de la siguiente forma: (a) Asistencia socio-comunitaria $(n=6)$, cuyo objetivo es la asistencia socio, sanitaria, legal a población en situación de desamparo/alto riesgo (deambulantes, sin techo, familias carenciadas, niños con enfermedades crónicas, ex presidiarios); (b) Arte, cultura, ocio y deporte $(n=3)$, cuyo objetivo es fomentar y difundir actividades culturales y deportivas; (c) Derecho, asesoramiento 
político-legal $(n=2)$, cuyo objetivo es la agrupación de inmigrantes y promoción de sus derechos; (d) Desarrollo ( $n=2$ ) cuyo objetivo es el desarrollo físico, intelectual, social de niños y jóvenes; (e) Hábitat, vivienda y medio ambiente $(n=2)$, cuyos objetivos son la promoción de la lucha por el acceso a la tierra, la vivienda y el hábitat, la protección, asistencia, recuperación y adopción de animales en situación de desamparo, accidentados y/o maltratados, así como la difusión y concientización de estas situaciones.

Se administró el Utrecht Work Engagement Scale (UWES-17) de Schaufeli, Salanova, González-Romá y Bakker (2001). Se trata de un cuestionario autoaplicado que incluye tres dimensiones o componentes constitutivos del engagement: vigor, dedicación, absorción. El UWES-17 puede utilizarse ya sea como instrumento unifactorial (que permite obtener un puntaje total único de engagement en el trabajo) o trifactorial (que permite obtener, además del puntaje total, un puntaje para cada una de sus dimensiones) dependiendo de los intereses del investigador. El instrumento presenta las siguientes cualidades psicométricas: (a) consistencia interna: es elevada (.93 a de Cronbach), (b) correlación interna: las tres dimensiones del UWES-17 están íntimamente relacionadas (exceden el $.65 \alpha$ de Cronbach), (c) estabilidad: los puntajes del UWES-17 son relativamente estables a través del tiempo (promedio de dos años para los coeficientes de vigor, dedicación y absorción) (BenevidesPereira, Fraiz de Camargo \& Porto-Martins, 2011).

Se procedió a la administración del instrumento UWES-17 a aquellos voluntarios que prestaron consentimiento informado respecto de su participación en la investigación. Los datos fueron cargados en matrices usando el programa SPSS (15.0). Posteriormente se procedió al análisis de descriptivos básicos y al cálculo de estadísticos no paramétricos para comparar grupos independientes según sean las variables sociodemográficas (Prueba de Mann-Withney y Prueba de Kruskal-Wallis).

Seguidamente se ofrecen los resultados, primero para el engagement considerado globalmente (puntaje total) y luego en relación con cada una de las dimensiones que lo componen. Luego se distribuyen casos según las variables sociodemográficas y los niveles de engagement. Para finalizar, se aplican pruebas no paramétricas para detectar niveles críticos de asociación.

\section{Niveles de engagement en ambos grupos de voluntarios}

En ambos grupos de voluntarios el 50\% o más presentan niveles altos de engagement. A su vez, el grupo de voluntarios universitarios acumula más del $40 \%$ en los niveles medios de engagement. Los niveles bajos y muy altos están presentes en ambos grupos, pero con porcentajes inferiores al $10 \%$ en los voluntarios de organizaciones asociativas y con

\footnotetext{
“Visión de Futuro" Año 17, Volumen N² 24 N¹, Enero - Junio 2020 - Pág. 131 - 148

URL de la Revista: http://visiondefuturo.fce.unam.edu.ar/index.php/visiondefuturo/index

URL del Documento: https://visiondefuturo.fce.unam.edu.ar/index.php/visiondefuturo/issue/view/17

ISSN 1668 - 8708 - Versión en Línea

E-mail: revistacientifica@fce.unam.edu.ar
} 
porcentajes inferiores al $5 \%$ en voluntarios universitarios.

Porcentajes cercanos al $50 \%$ son acumulados por voluntarios con niveles medios de absorción en ambos grupos. Le siguen los niveles altos de absorción que acumulan cerca del $35 \%$ de los voluntarios en ambos grupos. En voluntarios universitarios no se encontraron casos extremos con niveles muy altos o muy bajos en esta escala.

En relación con los niveles de dedicación, los porcentajes más elevados se acumulan en los niveles medio, alto y muy alto para ambos grupos. Los voluntarios de organizaciones asociativas acumulan más del $30 \%$ de sus voluntarios en los niveles muy altos de dedicación y los voluntarios universitarios acumulan más del $40 \%$ en los niveles altos de la misma dimensión. En cuanto al nivel bajo de dedicación, son escasos los voluntarios que se posicionan en este nivel. En ambos grupos de voluntarios no se presentan casos con niveles muy bajos en esta escala.

En cuanto a los niveles de vigor, el nivel medio acumula más del $45 \%$ de los voluntarios universitarios, lo mismo ocurre con el nivel alto en el mismo grupo. Más del 35\% de los voluntarios de organizaciones asociativas se distribuyen en niveles altos de vigor y más del $25 \%$ de ellos se acumulan en los niveles muy altos de esta dimensión.

\section{Engagement y variables sociodemográficas}

A continuación, se presentan asociaciones entre los niveles de engagement (puntaje total) y las variables sociodemográficas (sexo, edad, nivel de instrucción/formación, antigüedad como voluntario).

Respecto al sexo, tanto voluntarios femeninos como masculinos se distribuyen mayormente en los niveles medio y alto de engagement. Sin embargo, el sexo masculino presenta más casos en niveles muy altos de engagement en relación con el sexo femenino. Por el contrario, el nivel bajo de engagement se observa sólo en voluntarios de sexo femenino (ver Figura 1).

Puede observarse que en todos los rangos de edades y en ambos grupos los voluntarios se concentran en los niveles medio y alto de engagement (ver Figura 2).

En la Figura 3 se advierte que en todos los niveles de instrucción/formación predomina el nivel de engagement alto, a excepción de los sujetos que poseen universitario incompleto en los que, si bien el nivel alto es elevado predomina el nivel medio de engagement. Los niveles universitario completo y universitario incompleto presentan sólo un caso en nivel muy alto de engagement y, a su vez, son los únicos en donde se presentan casos de nivel bajo. Por su parte, son 4 y 3 los casos de terciarios completo que se ubican en nivel alto y muy alto de engagement respectivamente. Los casos con nivel primario completo, si bien son escasos, se distribuyeron en su totalidad en niveles altos de engagement.

\footnotetext{
“Visión de Futuro" Año 17, Volumen Nº 24 Nº̂, Enero - Junio 2020 - Pág. 131 - 148

URL de la Revista: http://visiondefuturo.fce.unam.edu.ar/index.php/visiondefuturo/index

URL del Documento: https://visiondefuturo.fce.unam.edu.ar/index.php/visiondefuturo/issue/view/17

ISSN 1668 - 8708 - Versión en Línea

E-mail: revistacientifica@fce.unam.edu.ar
} 


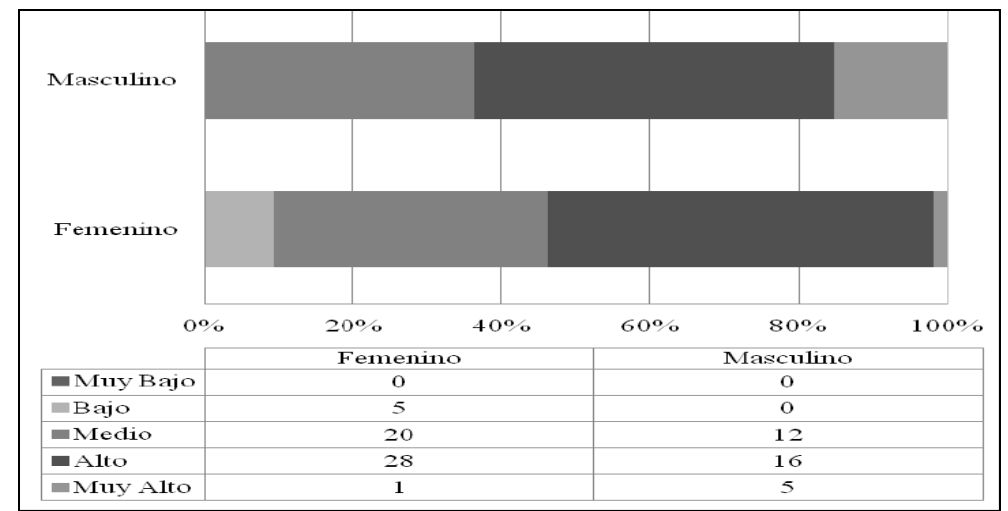

Figura $\mathrm{N}^{\circ} 1$. Distribución de voluntarios según nive I de engagement y sexo Fuente: Elaboración Propia

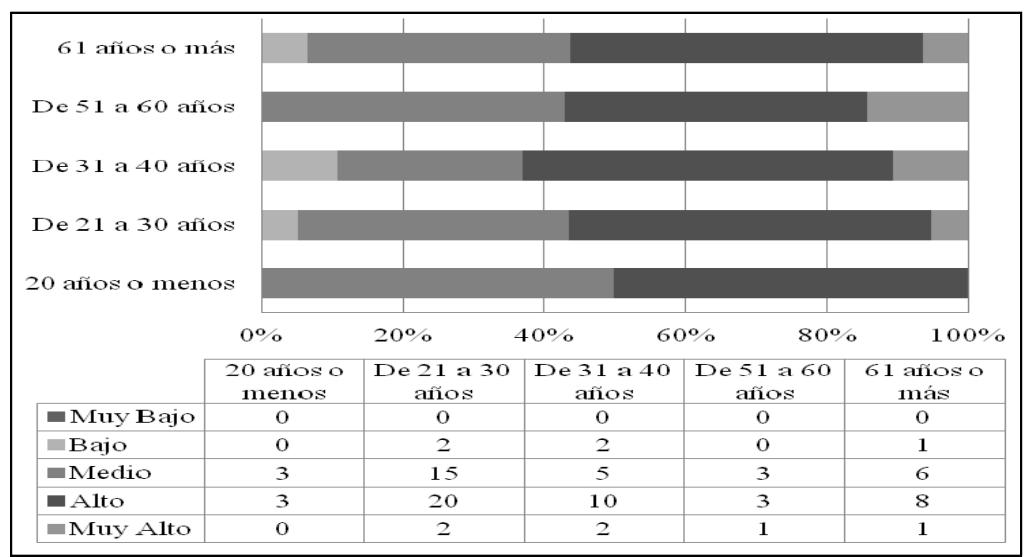

Figura №2. Distribución de voluntarios de ambos g rupos según nivel de engagement y edad Fuente: Elaboración Propia

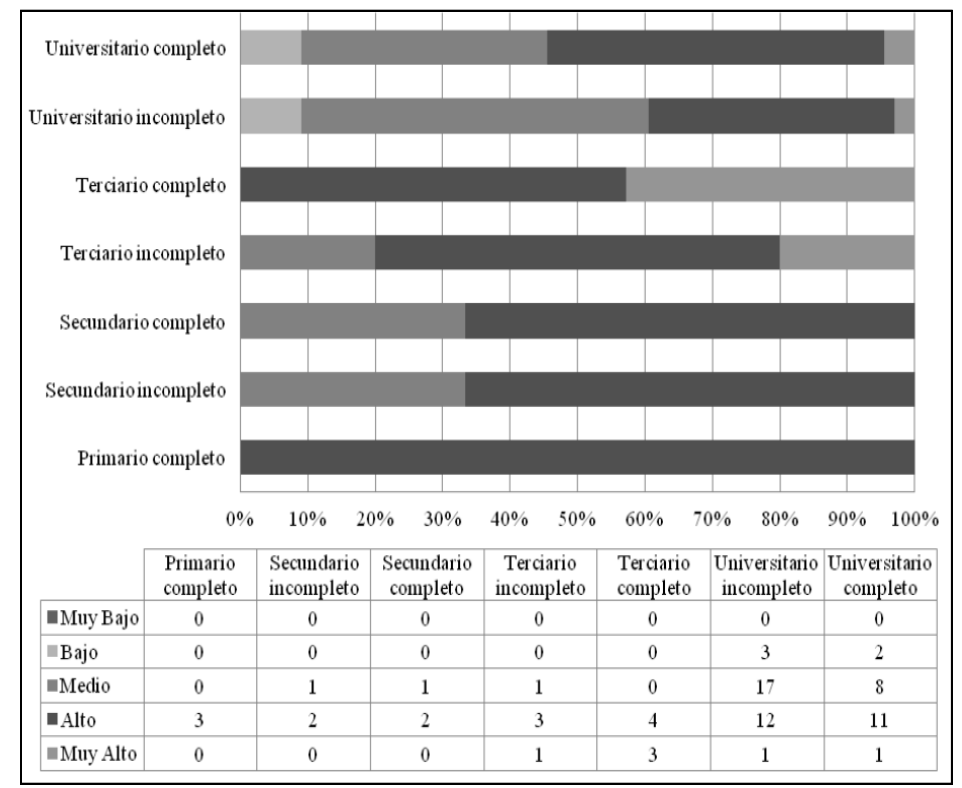

Figura $N^{\circ}$ 3. Distribución de voluntarios según nive I de engagement y nivel de instrucción/ formación Fuente: Elaboración Propia

“Visión de Futuro" Año 17, Volumen N² 24 N¹, Enero - Junio 2020 - Pág. 131 - 148

URL de la Revista: http://visiondefuturo.fce.unam.edu.ar/index.php/visiondefuturo/index

URL del Documento: https://visiondefuturo.fce.unam.edu.ar/index.php/visiondefuturo/issue/view/17

ISSN 1668 - 8708 - Versión en Línea

E-mail: revistacientifica@fce.unam.edu.ar 
En relación a la antigüedad como voluntario, los voluntarios con menos de 1 año de antigüedad se distribuyen entre los niveles medio y alto de forma equilibrada. Los voluntarios con una antigüedad de más de 1 año y hasta tres años, presentan el doble de casos en niveles altos respecto a los niveles medios. Lo mismo ocurre en voluntarios con hasta 5 años de antigüedad en donde los niveles altos duplican a los medios, aunque en este grupo son menos casos los que componen la muestra. Con más de 5 años de antigüedad, se distribuyen 3 casos en cada uno de los mencionados niveles (Ver Figura 4).

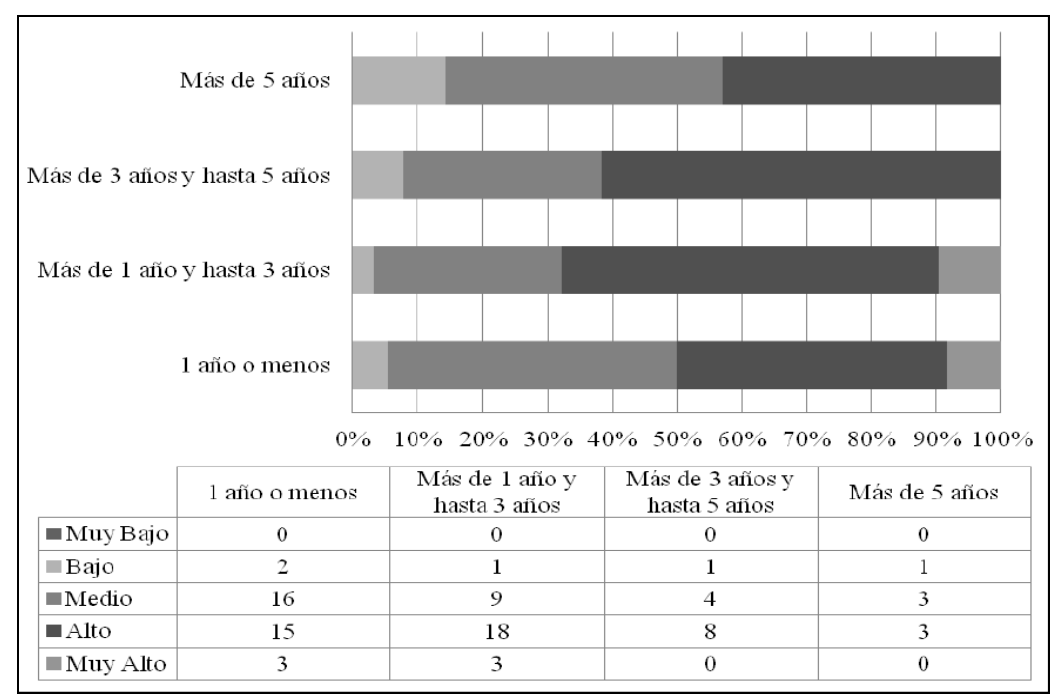

Figura $N^{\circ} 4$. Distribución de voluntarios según nive I de engagement y antigüedad en el proyecto/organización

Fuente: Elaboración Propia

\section{Análisis no paramétricos}

Para detectar niveles críticos de asociación entre variables sociodemográficas y escalas de engagement se aplicaron las pruebas $U$ de Mann-Withney para dos muestras independientes y Prueba de Kruskal-Wallis para varias muestras independientes.

La Tabla 1 contrasta grupos de voluntarios según sea el sexo aplicando la prueba $U$ de Mann-Withney. Se encontraron diferencias críticas en las dimensiones de engagement, dedicación y vigor. La Tabla 2 muestra el contraste de grupos según rangos de edad por medio de la prueba Kruskal-Wallis.

\footnotetext{
"Visión de Futuro" Año 17, Volumen Nº 24 N¹, Enero - Junio 2020 - Pág. 131 - 148

URL de la Revista: http://visiondefuturo.fce.unam.edu.ar/index.php/visiondefuturo/index

URL del Documento: https://visiondefuturo.fce.unam.edu.ar/index.php/visiondefuturo/issue/view/17

ISSN 1668 - 8708 - Versión en Línea 
Tabla $N^{\circ} 1$. Estadísticos de contraste según sexo de los voluntarios

\begin{tabular}{|c|c|c|c|c|}
\hline Estadísticos de contraste & Absorción & Dedicación & Vigor & Engagement \\
\hline U de Mann-Whitney & 753,000 & 666,500 & 645,500 & 710,500 \\
\hline W de Wilcoxon & 2238,000 & 2151,500 & 2130,500 & 2195,500 \\
\hline$Z$ & $-1,320$ & $-2,067$ & $-2,274$ & $-1,743$ \\
\hline Sig. asintót. (bilateral) &, 187 &, 039 &, 023 &, 081 \\
\hline
\end{tabular}

Nota. Variable de agrupación: Sexo. Prueba U de Mann-Whitney. Fuente: Elaboración Propia

Tabla $\mathbf{N}^{\circ}$ 2. Estadísticos de contraste según edad de los voluntarios

\begin{tabular}{|c|c|c|c|c|}
\hline $\begin{array}{c}\text { Estadísticos de } \\
\text { constraste }\end{array}$ & Absorción & Dedicación & Vigor & Engagement \\
\hline Chi-cuadrado & 1,803 & 4,719 & 1,872 &, 473 \\
\hline gl & 4 & 4 & 4 & 4 \\
\hline Sig. asintót. &, 772 &, 317 &, 759 &, 976 \\
\hline
\end{tabular}

Nota. Variable de agrupación: Edad. Prueba de Kruskal-Wallis. Fuente: Elaboración Propia

Tabla №3. Estadísticos de contraste según nivel d e instrucción/formación de los voluntarios

\begin{tabular}{|c|c|c|c|c|}
\hline $\begin{array}{c}\text { Estadísticos de } \\
\text { constraste }\end{array}$ & Absorción & Dedicación & Vigor & Engagement \\
\hline Chi-cuadrado & 12,537 & 10,027 & 23,849 & 17,033 \\
\hline gl & 6 & 6 & 6 & 6 \\
\hline Sig. asintót. &, 051 &, 124 &, 001 &, 009 \\
\hline
\end{tabular}

Nota. Variable de agrupación: Nivel de instrucción/ formación. Prueba de Kruskal-Wallis. Fuente: Elaboración Propia

La misma prueba se aplicó para contrastar grupos de voluntarios según nivel de instrucción/formación, encontrando niveles críticos en la dimensión vigor y en el puntaje total de engagement (Tabla 3). Para detectar entre qué niveles de instrucción se encuentran los valores significativos, se procedió a realizar una comparación dos a dos aplicando la prueba U de Mann-Withney (Tabla 4). Según la Tabla 4, se encontraron valores significativos entre varios de los niveles de instrucción/formación comparados dos a dos. La dimensión vigor es la que se evidencia como más crítica ya que muestra valores críticos en casi la totalidad de los grupos comparados. Luego sigue puntaje total de engagement sobre todo cuando se comparan grupos universitarios (incompletos /completos).

\footnotetext{
"Visión de Futuro" Año 17, Volumen No 24 N¹, Enero - Junio 2020 - Pág. 131 - 148

URL de la Revista: http://visiondefuturo.fce.unam.edu.ar/index.php/visiondefuturo/index

URL del Documento: https://visiondefuturo.fce.unam.edu.ar/index.php/visiondefuturo/issue/view/17

ISSN 1668 - 8708 - Versión en Línea

E-mail: revistacientifica@fce.unam.edu.ar
} 
Tabla N4. Estadísticos de contraste según nivel de instrucción/formación de los voluntarios

\begin{tabular}{|l|c|c|}
\hline Niveles de instrucción/formación comparados & Vigor & Engagement \\
\hline Primario completo y secundario incompleto &, 036 &, 232 \\
\hline Primario completo y universitario incompleto &, 015 &, 072 \\
\hline Primario completo y universitario completo &, 047 &, 205 \\
\hline Secundario incompleto y secundario completo &, 036 &, 939 \\
\hline Secundario incompleto y universitario incompleto &, 015 &, 375 \\
\hline Secundario incompleto y universitario completo &, 047 &, 711 \\
\hline Secundario completo y terciario completo &, 003 &, 007 \\
\hline Terciario incompleto y universitario incompleto &, 048 &, 062 \\
\hline Terciario completo y universitario incompleto &, 000 &, 001 \\
\hline Terciario completo y universitario completo &, 003 &, 006 \\
\hline
\end{tabular}

Nota. Sólo constan en la tabla las comparaciones entre niveles de instrucción/ formación que han presentado al menos un valor crítico en la dimensión vigor y engagement (puntaje total).

Fuente: Elaboración Propia

Seguidamente, se aplicó la prueba Kruskal-Wallis para contrastar voluntarios según su antigüedad como tal no encontrando niveles críticos (Tabla 5).

Tabla $\mathbf{N}^{\circ} 5$. Estadísticos de contraste según antigüe dad como voluntario

\begin{tabular}{|c|c|c|c|c|}
\hline $\begin{array}{c}\text { Estadísticos de } \\
\text { constraste }\end{array}$ & Absorción & Dedicación & Vigor & Engagement \\
\hline Chi-cuadrado & 1,373 &, 081 & 5,951 & 2,986 \\
\hline gl & 3 & 3 & 3 & 3 \\
\hline Sig. asintót. &, 712 &, 994 &, 114 &, 394 \\
\hline
\end{tabular}

Nota. Variable de agrupación: Antigüedad en la organización. Prueba de Kruskal-Wallis. Fuente: Elaboración Propia

Por fin, se calcularon estadísticos de contraste ( $U$ de Mann-Whitney) según sea el tipo de voluntariado (voluntariado universitario y voluntariado de organizaciones asociativas), encontrando que no habría niveles críticos en las muestras consideradas (Tabla 6).

Tabla $N^{\circ}$ 6. Estadísticos de contraste según tipo de voluntariado

\begin{tabular}{|c|c|c|c|c|}
\hline Estadísticos de constraste & Absorción & Dedicación & Vigor & Engagement \\
\hline U de Mann-Whitney & 759,500 & 847,500 & 714,500 & 823,000 \\
\hline W de Wilcoxon & 1255,500 & 1343,500 & 1210,500 & 1319,000 \\
\hline$Z$ & $-1,051$ &,- 191 & $-1,441$ &,- 440 \\
\hline Sig. asintót. (bilateral) &, 293 &, 848 &, 150 &, 660 \\
\hline
\end{tabular}

Nota. Variable de agrupación: Tipo de voluntariado. Prueba U de Mann-Whitney.

Fuente: Elaboración Propia

\footnotetext{
"Visión de Futuro" Año 17, Volumen No 24 N¹, Enero - Junio 2020 - Pág. 131 - 148

URL de la Revista: http://visiondefuturo.fce.unam.edu.ar/index.php/visiondefuturo/index

URL del Documento: https://visiondefuturo.fce.unam.edu.ar/index.php/visiondefuturo/issue/view/17

ISSN 1668 - 8708 - Versión en Línea

E-mail: revistacientifica@fce.unam.edu.ar
} 


\section{CONCLUSIÓN}

Los resultados analizados permiten observar que ambos tipos de voluntariado se comportan de manera similar en cuanto a nivel de engagement (puntaje total) ya que ambos grupos concentran un $90 \%$ de voluntarios en niveles medio y alto de engagement. $\mathrm{A}$ continuación, se discuten los resultados del análisis por dimensión de engagement (absorción, vigor y dedicación).

La absorción, como fue señalado al inicio, indica concentración, sensación de disfrute, realización y dificultades para desligarse del trabajo. Estas características se dan, en la mayoría de los voluntarios, en los niveles medio y alto siendo considerablemente mayor el nivel medio en relación al alto. Los niveles se distribuyen de manera similar para ambos grupos de voluntariado. En cuanto a la dimensión de dedicación (concentración e implicación en el trabajo), ambos tipos de voluntariado califican con los dos niveles superiores alto y muy alto. El vigor que indica activación, esfuerzo y persistencia es más elevado en voluntarios de organizaciones asociativas que entre los voluntarios universitarios.

Los niveles bajo y muy bajo tienen reducida presencia para cada una de las dimensiones. Esto sugeriría que la actividad de voluntariado se asocia a niveles de engagement medios o superiores en todas sus dimensiones.

Los estadísticos de contraste calculados (prueba $U$ de Mann-Whitney) muestran que no habría diferencias significativas comparando ambos grupos por dimensión de engagement.

Específicamente, el análisis no paramétrico del engagement y sus dimensiones en relación con distintas variables sociodemográficas permite decir que:

(a) En relación con el sexo habría diferencias significativas en la dimensión dedicación y vigor, estos resultados contrastan con los de otras investigaciones que no hallaron dichas diferencias por sexo (Chiang, Núñez, Martín y Salazar, 2010). Si bien son pocos los casos considerados, los niveles más altos de engagement (puntaje total), se encontraron mayormente en voluntarios masculinos y en los niveles más bajos de engagement se encontraron sólo en voluntarios de sexo femenino. Este resultado también apoya los hallazgos de Benevides-Pereira, Fraiz de Camargo y Porto-Martins (2011).

(b) En todos los rangos de edad considerados el engagement se distribuye de manera similar predominando los niveles medio y alto. Al agrupar por la variable edad, no se presentan niveles diferenciales de engagement (ni por puntaje total ni por dimensiones). Coincidimos en este punto con los resultados de Benevides-

\footnotetext{
“Visión de Futuro" Año 17, Volumen No 24 N¹, Enero - Junio 2020 - Pág. 131 - 148

URL de la Revista: http://visiondefuturo.fce.unam.edu.ar/index.php/visiondefuturo/index

URL del Documento: https://visiondefuturo.fce.unam.edu.ar/index.php/visiondefuturo/issue/view/17

ISSN 1668 - 8708 - Versión en Línea

E-mail: revistacientifica@fce.unam.edu.ar
} 
Pereira, Fraiz de Camargo y Porto-Martins (2011) y Chiang, Núñez, Martín y Salazar (2010). De todas formas, cabe mencionar que las submuestras que se incluyen en cada rango de edad son muy reducidas y que futuros estudios podrían contrastar estos resultados ampliando las mismas.

(c) En cuanto a los niveles de instrucción/formación, se encontraron diferencias críticas en la dimensión vigor y engagement (puntaje total). Las diferencias críticas aparecen sobre todo al comparar niveles de instrucción/formación terciarios con universitarios, sean éstos completos o incompletos. Se considera deseable aumentar el número de participantes en futuras investigaciones para poner a prueba la hipótesis que vincula engagement a nivel de instrucción/ formación.

(d) Analizando la antigüedad como voluntario, no se encontraron niveles significativos que diferencien los grupos por antigüedad.

(e) Finalmente, tampoco se encontraron niveles críticos comparando grupos por tipo de voluntariado (universitario / de organizaciones asociativas).

Considerando el primer objetivo particular: describir los niveles de engagement en ambos grupos de voluntarios, la investigación ha permitido constatar que no existen diferencias significativas en los niveles de engagement (puntaje total) ni en las dimensiones absorción, vigor y dedicación entre grupos de voluntariado universitario y voluntariado de organizaciones asociativas. La gran mayoría de los voluntarios que participaron de este estudio han presentado niveles medios y altos de engagement (puntaje total).

Se encontraron diferencias significativas al contrastar grupos según sexo en las dimensiones dedicación y vigor. Esto significa que habría diferencias entre voluntarios masculinos y voluntarias femeninas en cuanto a niveles de entusiasmo e implicación en el trabajo y niveles de activación, esfuerzo y persistencia. En cuanto a nivel de instrucción/formación de los voluntarios se encontraron niveles críticos en la dimensión vigor y engagement (puntaje total).

No se han encontrado niveles críticos en grupos según edad ni según antigüedad en la actividad de voluntariado.

Cabe mencionar una serie de limitaciones presentes en el estudio. En primera instancia el número de participantes siendo que se trabajó con una muestra intencional. Este estudio constituye un primer acercamiento a la temática, desde el grupo de investigación se continuará trabajando con voluntarios de diferente pertenencia institucional en el marco de las problemáticas sociales actuales de nuestro país que requieren primordialmente de actitudes de responsabilidad y compromiso social. Otra limitación radica en el instrumento de recolección de datos, el UWES-17 ha sido aplicado de manera auto-administrada, futuras 
investigaciones deberían considerar triangular resultados del UWES-17 con otros obtenidos por medio de metodologías de carácter más cualitativo.

Esta investigación tiene impacto directo en los procesos de toma de decisiones en relación a la formulación de criterios de selección en convocatorias de voluntariado (tanto universitario como en organizaciones del Tercer Sector), considerando así perfiles de voluntarios engaged a la hora de seleccionar los equipos de trabajo. En relación a ello, en esta investigación no se han encontrado diferencias críticas por grupos de edad ni por antigüedad como voluntario, lo cual podría impactar a la hora de atenuar restricciones por edad o experiencia previa que en ocasiones se sostienen en las convocatorias de voluntariado. Sí se encontraron algunas diferencias críticas por sexo y por nivel de instrucción/formación, aunque deberían contrastarse en muestras más amplias.

Por otro lado, los resultados de esta investigación también impactan en el diseño de procesos de capacitación y desarrollo de voluntarios que puedan orientarse al fortalecimiento de competencias psicosociales vinculadas a la tridimensionalidad del engagement.

En síntesis, esta investigación muestra la alta importancia de la variable engagement y sus dimensiones (vigor, dedicación y absorción) para el trabajo voluntario tanto en organizaciones no gubernamentales como en proyectos universitarios. No se han encontrado investigaciones previas que estudien esta variable y sus dimensiones en contextos de voluntariado. Desde una universidad socialmente comprometida es relevante promover un voluntariado que fortalezca competencias psicosociales para abordar las actuales problemáticas y para promover una sociedad solidaria, participativa y comprometida.

\section{REFERENCIAS}

Arias Careaga, S. (2008). Voluntariado universitario. Guía para su gestión en las universidades madrileñas. Madrid: Dirección General de Voluntariado y Promoción Social de la Comunidad de Madrid.

Benevides-Pereira, A., Fraiz de Camargo, D. y Porto-Martins, P. (2011) Uwes: Utrech Work Engagement Scale. Holanda: Utrecht University.

Blanch, J.M.; Sahagún, M. y Cervantes, G. (2010). Estructura factorial del Cuestionario de Condiciones de Trabajo Factor Structure of Working Conditions Questionnaire. Revista de Psicología del Trabajo y de las Organizaciones, 26(3), 175-189.

Cabezas Gonzáles, M. (2001). Reflexiones críticas sobre el voluntariado. Papeles 
Salmantinos de educación, 0, 12-32.

Chacón, F. \& Vecina, M.L. (2002). Gestión del voluntariado. Madrid: Síntesis.

Chiang, M., Núñez, A., Martín, M. y Salazar, M. (2010). Compromiso del trabajador hacia su organización y la relación con el clima organizacional: un análisis de género y edad. Panorama Socioeconómico, 28(40), 90-100.

Clary, G. \& Snyder, M. (1999). The Motivations to Volunteer: Theoretical and Practical Considerations. Psychological Science, 8(5), 156-159.

Cruz Roja Española (2002). Formación básica institucional. Manual del voluntariado. Madrid: Cruz Roja Española.

Dávila de León, M. y Chacón Fuertes, F. (2004). Factores psicosociales y tipo de voluntariado. Psicothema, 16(4), 639-645.

Davis Smith, J. (2002, septiembre). Civic Service in Western Europe. Presentado en The Global Service Institute Research Conference, Toward a Global Research Agenda on Civic Service, St. Louis.

Díez, M. y Dolan, S. (2008). Burnout vs. vigor profesional: Análisis configuracional de las características sociolaborales e individuales protectoras en médicos catalanes. Revista de Psiquiatría del Uruguay, 72(2), 169-185.

Estébanez, P. (2005). Las Organizaciones No Gubernamentales (ONG). En P. Estébanez (Ed.), Medicina Humanitaria (pp. 125-136). Madrid: Díaz de Santos.

García-Renedo, M.; Llorens, S.; Cifre, E. y Salanova, M. (2006). Antecedentes afectivos de la auto-eficacia docente: un modelo de relaciones estructurales. Revista de Educación, 0(339), 387-400.

González-Roma, V.; Schaufeli, W.B; Bakker, A.B. \& Lloret, S. (2006). Burnout and work Engagement: Independent factors or opposite poles? Journal of Vocational Behavior, 68, 165-174.

Gutierrez Resa, A. (1997). Acción social no gubernamental. Análisis y reflexiones sobre las relaciones voluntarias. Valencia: Tirant lo Blanch.

Hustinx, L.,Vanhove, T., Declercq, A., Hermans K. \& Lammertyn, F. (2005). Bifurcated Commitment, Priorities and Social Contagion: The Dynamics and Correlates of Volunteering within a University Student Population. British Journal of Sociology of Education, 26, 523-538.

Lisbona, A., Morales, J. \& Palací, F. (2009) El engagement como resultado de la socialización organizacional. International Journal of Psychology and Psychological Therapy, 9(1), 89-100.

Manzano, G. (2002). Burnout y engagement en un colectivo pre-profesional estudiantes universitarios. Boletín de Psicología, 74, 79-102 
Maslach, C. y Leiter, M. P. (1997). The Truth about Burnout. San Francisco: Jossey-Bass.

Roitter, M. (2001). Apuntes para contribuir a (des)cifrar el sector no lucrativo argentino en el contexto latinoamericano. Ponencia presentada en Tercer Encuentro de la Red Latinoamericana de ISTR. Buenos aires.

Salanova, M., Agut, S. y Peiró, J.M. (2005). Linking Organizational Resources and Work Engagement to Employee Performance and Customer ILyalty: The Mediating Role of Service Climate. Journal of Applied Psychology, 90, 1217-1227

Salanova, M., Schaufeli, W. B., Llorens, S., Peiró, J. M. y Grau, R. (2000). Desde el "Burnout" al "Engagement": ¿Una nueva perspectiva? Revista de Psicología del Trabajo y las Organizaciones, 16(2), 117-134

Schaufeli, W.B., Salanova, M., González-Romá, V., \& Bakker, A. (2001). UWES: Utrecht Work Engagement Scale, Preliminary Manual. Holanda: Utrecht University.

Schaufeli, W.B., Martínez, I., Marques Pinto, A. Salanova, M. y Bakker, A.B. (2002). Burnout and Engagement in university students: A cross national study. Journal of CrossCultural Psychology, 33, 464-481

Seligman, M. E. P. \& Csikszentmihalyi, M. (2000). Positive Psychology: An Introduction, American Psychologist, 55, 3-6.

Tang, F., McBride, A. M. y Sherraden, M. (2003). Toward Measurement of Civic Service (CSD Report 03-33). St. Louis: Washington University, Center for Social Development.

Tapia, M. N. (2007). Voluntariado, sociedad civil y democracia. Una mirada latinoamericana. Servicio Cívico y Voluntariado en Latinoamérica y el Caribe, 2.

Tapia, M. N. (2010, noviembre). Fortalezas y debilidades de la labor del voluntariado universitario en América Latina. Presentada en Seminario Internacional Políticas e Instrumentos de Gestión para Potenciar el Voluntariado Universitario, Madrid.

Vecina, M. (2001). Factores psicosociales que influyen en la permanencia del voluntariado. Tesis doctoral no publicada, Universidad Complutense de Madrid. España.

Vecina, M., Chacon, F. y Sueiro, M. (2010). Differences and Similarities among Volunteers who Drop out During the first Year and Volunteers who Continue after eight Years. Spanish J ournal of Psychology, 13(1), 343-352.

Vecina, M., Chacón, F., Sueiro, M. y Barrón, A. (2012). Volunteer Engagement: Does Engagement Predict the Degree of Satisfaction among New Volunteers and the Commitment of Those who have been Active Longer? Applied Psychology: An International Research, 61, 130-148.

\footnotetext{
“Visión de Futuro" Año 17, Volumen No 24 N¹, Enero - Junio 2020 - Pág. 131 - 148

URL de la Revista: http://visiondefuturo.fce.unam.edu.ar/index.php/visiondefuturo/index

URL del Documento: https://visiondefuturo.fce.unam.edu.ar/index.php/visiondefuturo/issue/view/17

ISSN 1668 - 8708 - Versión en Línea 


\section{RESUMEN BIOGRÁFICO}

\section{Yamila Fernanda Silva Peralta}

Lic. en Psicología (Fac. Psicología-UNMDP). Magister Psicología del Trabajo de las Organizaciones y de los RRHH (Univ. Barcelona). Magister en Ciencias Humanas y Sociales (Univ. Paris 5). Doctora en Psicología (Univ. de. Barcelona). Docente de grado/posgrado, Investigadora CONICET - Facultad Psicología-UNMDP. Directora tesistas de grado/posgrado y de proyectos de investigación y extensión universitaria. Asesora Área de Relaciones Internacionales de UNMDP.

\section{Sergio Gastón Arias}

Es Lic. en Psicología (Fac. Psicología, UNMDP). Ha desarrollado su tesis de grado sobre desarrollo de competencias en voluntarios.

\section{Lorena Verónica Caracciolo}

Es Lic. en Psicología (Fac. Psicología, UNMDP). Ha desarrollado su tesis de grado sobre desarrollo de competencias en voluntarios.

\section{Juan Pablo Vega}

Es Lic. en Psicología (Fac. Psicología, UNMDP). Ha desarrollado su tesis de grado sobre desarrollo de competencias en voluntarios.

\section{María Emilia Rompato}

Es Lic. en Sociología (Fac. Humanidades, UNMDP). Es tesista doctoral de la Fac. de PsicologíaUNMDP y Becaria de Investigación de la UNMDP. Investiga sobre competencias emprendedoras psicosociales en el marco de la Educación Superior. 